\title{
REVIEW OF 3D GIS DATA FUSION METHODS AND PROGRESS
}

\author{
Wei Hua ${ }^{1}$, Miaole Hou ${ }^{1,2,3} *$, Yungang $\mathrm{Hu}^{1,2,3 *}$ \\ ${ }^{1}$ School of Geomatics and Urban Spatial Informatics, Beijing University of Civil Engineering and Architecture, No.15Yongyuan \\ Road, Daxing District, Beijing, 102616-(huawei, houmiaole, hyg)@ bucea.edu.cn \\ ${ }^{2}$ Beijing Key Laboratory For Architectural Heritage Fine Reconstruction \& Health Monitoring, No.15Yongyuan Road, Daxing \\ District, Beijing, 102616 \\ ${ }^{3}$ Engineering Research Center of Representative Building and Architectural Heritage Database, Ministry of Education, \\ No.15Yongyuan Road, Daxing District, Beijing, 102616
}

\section{Commission III, WG III/6}

KEY WORDS: Data Fusion, Terrain Model, Ground Object Model, Triangulated Irregular Network, Regular Square Grid

\begin{abstract}
:
3D data fusion is a research hotspot in the field of computer vision and fine mapping, and plays an important role in fine measurement, risk monitoring, data display and other processes. At present, the research of 3D data fusion in the field of Surveying and mapping focuses on the 3D model fusion of terrain and ground objects. This paper summarizes the basic methods of 3D data fusion of terrain and ground objects in recent years, and classified the data structure and the establishment method of 3D model, and some of the most widely used fusion methods are analysed and commented.
\end{abstract}

\section{INTRODUCTION}

3D GIS is a new technology developed based on 2D GIS, it breaks through the spatial information in the 2D map plane monotonic performance constraints, to achieve high precision modelling, 3D integration analysis, accurate measurement, integrated management and real-time visualization analysis. There are more extensive applications in the fields of Intelligent City, construction engineering, examination of ancient literature and so on. Considering the production difficulty, efficiency and cost of 3D models, terrain and ground object models are often constructed by different methods in 3D GIS, so they have different data structures and characteristics. Taking 3D terrain model as an example, according to the different types of model grid, three-dimensional GIS terrain has a Regular Square Grid (RSG) model and a Triangulated Irregular Network (TIN) model (Gong, 2006). The method of 3D object modelling is more diverse. It can be obtained by three dimensional laser scanning, oblique photogrammetry, and professional 3D modelling software or 3D GIS system. In 3D GIS, in order to satisfy the requirements of terrain and ground object modelling in accuracy, vision and query, we need to merge the terrain and terrain 3D models. Through the paste and repair of the 3D model (Chen, 2016), the effect of smooth boundary and texture splicing is achieved, and finally the new 3D digital model is fused.

The terrain model and object model fusion earlier research began in 1995, Michael F. Polls had vector road data through the geometric embedding method into terrain grid (Polis M F, Gifford S J, Jr M K, 1995), but the road model itself does not have fluctuant form, just as the existence of vector data, and does not address the dynamic change of terrain model LOD. In recent years, the main problem for $3 \mathrm{D}$ model fusion is in $3 \mathrm{D}$
GIS system, the ground object models are often established based on the horizontal datum, while the regular grid terrain is built on the basis of the undulating features of the DEM, resulting in a mismatch between the two models. Typically, the terrain model is suspended above the terrain model or the ground model part is hidden under the terrain model (Figure 1). In order to solve this problem, according to the difference of terrain and grid types, HU Lujing, Liu Xiaobiao, Li Qiang and other scholars divide the three dimensional GIS terrain into regular grid terrain and irregular triangulated network terrain (Hu, 2013; Liu, 2014; Li, 2008), and study the corresponding model fusion respectively. There are also some scholars have classified the object model into two kinds which are independent and dependent on the terrain Grid Terrain grid, and further subdivided into point objects, banded terrain and areal ground features, is proposed based on Terrain Reconstruction and transformation based on different feature fusion method, representative Wang Guangxia, Liu Ning, WanGang Based on the integration of research Based on the original three dimensional model fusion, to help users browse with confidence (Wang, 2005; Liu, 2004; Wan, 2001), Anupam Agrawal, Angelika Weber, Michael F. Polls, Chen Guojun add the research of multi-scale terrain model fusion, and improve the research of LOD display after 3D data fusion(Agrawal, 2006; Michael F, 1995; Chen, 2009).

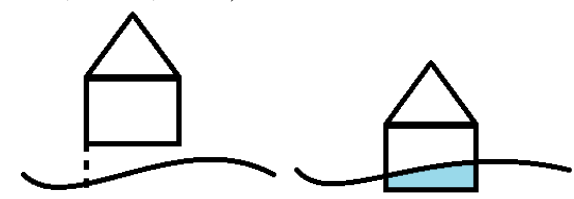

Figure 1. None matching of terrain model with object model

* Corresponding author. E-mail addresses: houmiaole@bucea.edu.cn (Miaole Hou), hyg@ bucea.edu.cn (Yungang Hu). 
In this paper, the fusion method of terrain model and ground object model is systematically classified, this paper also summarizes and reviews all kinds of 3D data fusion methods in recent years, and discusses the problems and prospects for future development based on tilting photogrammetry model fusion.

\section{CONSTRUCTION AND EXPRESSION OF 3D MODEL}

There are four ways to build 3D models. One is based on the active collection of $3 \mathrm{D}$ point cloud data, and then to build $3 \mathrm{D}$ models through denoising, patching, surface fitting and so on. The second is the passive depth reconstruction based on the oblique photograph and the establishment of a 3D model. The third is to use 3D modelling software, such as 3D MAX, Maya and other software, to make 3D model through given geometric information and topological information. The last one is to use a 3D GIS system to design a 3D model with spatial topology in City Engine or ArcGIS software.

In the 3D GIS system, the terrain model is generally expressed in two ways, one is the Regular square grid model, and the other is the Triangulated irregular network model. Regular square grid model is one of the main ways of terrain representation. Most of them use the fixed square grid to express terrain information. Grid coordinates express spatial location information, and grid data value is elevation value (Figure 2). The Regular square grid model has the advantage of simple data structure. Because grid size is fixed, it can use two dimensional matrix to store the elevation of each point. In computer language, it is equivalent to an array, easy to use and easy to manage. The shortcoming is the large amount of storage, a large amount of data redundancy in the simple terrain, and low resolution in the complex terrain.

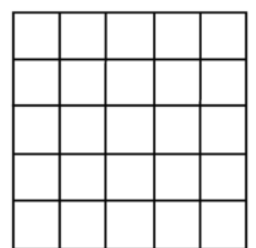

Figure 2. Regular square grid model

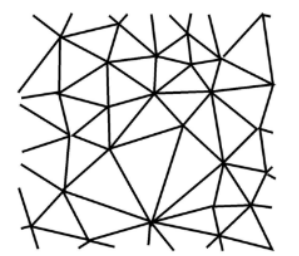

Figure 3.Triangulated irregular network model

The Triangulated irregular network model is also one of the ways of terrain representation. It generates continuous triangular surfaces from irregular data points to approximate the surface of the terrain (Figure 3), which can restore the slight fluctuations of the surface to the greatest extent and take good account of the terrain features. So it is also called surface data structure. Compared to the Regular square grid model, the vertex distribution in the Irregular Triangulation model is arbitrary. Therefore, the Irregular Triangulation terrain model can simulate the complex terrain surface more accurately, with high accuracy, small data redundancy and high storage efficiency, which is suitable for multi-level expression. But the data structure of Triangulated irregular network model is complex, the storage is large, the use and management are complex, the algorithm is complex, and the curved surface cannot be expressed.

\section{METHOD OF 3D MODEL FUSION}

\subsection{Fusion of Terrain Model and Ground Object Model Independent of Terrain}

The fusion of terrain independent terrain models (such as vegetation and building models) can be simplified as spatial location registration. There are two main types of fusion: One method is that the terrain model can be directly registered and superimposed without modifying the terrain model. This method is simple, but after the fusion, it is very easy to appear the hole in the model. Another method is to modify the terrain mesh model to adapt the boundary to the ground object model in order to achieve the close match of the model after the fusion. Because of the high accuracy of the second method for model fusion, the method of adapting the ground object model adaptable to the terrain model is currently with appropriate modification of the terrain model.

3.1.1 Accurate Matching Fusion Method: In the fusion of the independent ground object model and the rule grid terrain model, the typical architecture model is fused with the terrain model. Transformation of terrain model or transformation of terrain model to achieve model fusion with each other. This method extends the building model down to the lowest level of the terrain covered by the model, or achieves the fusion effect by comparing the original terrain with the increase or decrease of the bottom of the building. The essence of this method is the precise matching of the terrain model and the terrain model in the space position, the adaptation of the ground object model to the integration of terrain model by proper modification of terrain model. Wang Aiai, Zhang Jin adopts two different fusion methods (Wang, 2012): one is the transformation of object model: through the following structural feature model datum model to complete the integration with the terrain model; the other is the transformation of the terrain model: the object model in the coverage area of the flat terrain and buildings will be unified, the bottom boundary of the grid split into terrain grid model fusion. In 2003, Song Hanchen and others put forward the four point matching method (Song, 2003), projecting the selected key points to the terrain surface, and displaying the real-time gesture of the model in view of the additional information such as the location of the view and the adjustment matrix, so as to ensure the fusion of visual models. But this method is actually a visual model matching, and the 3D model is not actually fused. Based on this integration, Arne Schilling 0 and so on have studied and implemented the transformation of complex 3D geographical elements into different LOD detail level visual display under virtual reality server (Arne Schilling, 2003), and then converted to LOD model display with limited range. Chen Guojun and Chen Qinggang expand the resolution of cell transformation (Chen, 2009), describe the feature contours with more precise rules triangle, and combine the view related LOD simplification technology to achieve the fusion of ground object model and terrain model.

This kind of fusion method has changed the original 3D model, and the fusion model will have some influence on the measurement accuracy. For data fusion between 3D models of different resolution, whether this method is still effective is expected to be further studied by relevant scholars. 
3.1.2 Mesh Vertex Pre Fusion Method: Vertex pre fusion is a pre ground object model and terrain model is the mesh vertex or point cloud to express the way, using the method of point cloud sampling or constraint region deletion mesh vertex fusion object model and terrain model in point cloud level, finally the grid vertex integration good constructing triangulated irregular network (TIN) to achieve the fusion effect.

Scholar Li Lin first unified the coordinate system, and then used the altitude data of the bottom plane of the pre fusion ground object model as a part of the topographic elevation data $(\mathrm{Li}$, 2011). At last, he removed the elevation data of the fusion of terrain data and 3D digital models in the bottom plane of the pre fusion ground object model, and then used the Delaunay triangulation algorithm to generate the TIN model to simulate the terrain. In this way, the fusion method of 3D terrain model and 3D terrain model is realized. Geng Zhongyuan adopted the way of building up the fusion buffer area (Geng, 2016), and using the tilting photography topographic model instead of the original terrain model in the buffer area to achieve the integration of the models. Firstly, the ground object model with a small amount of terrain data is separated from oblique photography, in which a small amount of terrain data is represented by point cloud, and the ground object model is represented by Irregular Triangulation Network (TIN). In the integration of the buffer zone, the establishment of a tilt photography terrain point cloud model instead of the original terrain point cloud model, and in accordance with the two kinds of terrain model different point cloud density, on the basis of arithmetic sequence acquisition sampling interval, the sampling density gradually. Finally, the Delaunay triangulation algorithm is used to construct the topographic point cloud model with good integration and construct the terrain model based on the Irregular Triangulation Network (TIN), so as to achieve the integration of the tilted photograph object model and the terrain model.

Using the fusion point cloud data to regenerate the irregular triangulation net again, and the fusion of the model is the main feature of the mesh vertex fusion. But because of the original point cloud data was deleted in the process of data fusion, triangulated irregular network will also receive the corresponding effect of re generated. Especially the 3D model with real texture photogrammetry based on the tilt, due to changes in model after fusion re generate the independent model compared to the previous triangulation irregular triangle net irregular texture mapping rules, the original is no longer applicable to the three dimensional model after fusion, so there will be a blank, texture distortion and other issues. This is also the rapid development of UAV aerial survey technology. Today, based on UAV aerial photogrammetry, we need further research on the process of $3 \mathrm{D}$ model fusion with real texture.

\subsection{Fusion of Terrain Model and Ground Object Model Dependent of Terrain}

Terrain dependent terrain can be regarded as a part of the terrain. Most of them are embedded in the terrain models. They can be differentiated from the terrain models, and are closely related to the undulating trend of the terrain. The influence of the terrain models should be fully considered when merging.

The objects of this type of model include roads, rivers, lakes, large areas of vegetation, etc. The process of fusion and construction of this kind of ground object model and terrain model is actually the process of triangulation of polygons.

According to the differences of the geometry and the boundary range of elevation values, ground object model dependent of terrain can be divided into two types:
1) The elevation values of the surface distribution and the boundary range are the same (such as lakes, large area vegetation, etc.).

2) Different elevation values within the boundaries of their own boundaries and zonal distribution (such as roads, rivers, etc.).

3.2.1 DEM Grid Cutting Fusion Method: For the first surface distribution model, the method of fusion is based on the projection of DEM. First, the average value of the ground elevation in the area of the surface of the surface is used as the elevation value of the area, and the polygon of the area is triangulated. Then, the polygon of the area is projected onto the two-dimensional plane and intersected with the plane projection of the terrain model DEM grid, and the elevation value of the intersection point is assigned to H. Finally, the new DEM mesh is triangulated, thus the fusion of surface ground object model and terrain model is completed.

Aiming at the second kinds of stripped part of the ground object model, because of the greater influence on the terrain model, the method of fusion is triangulation based on the intersection point between the ground object model and the terrain model. Taking the road model as an example, with the road model closed polygonal boundary and the DEM grid intersection point, and all the intersection into the DEM grid, and then the DEM triangle grid intersection points form a polygon. Finally, the dissection of the existing data in the DEM grid and the simple section of the road model can be combined with the terrain model. In 2005, Wang Guangxia and others compared the terrain model with the original terrain model on the basis of the fusion (Wang, 2005), and determined the accuracy evaluation of the fusion algorithm. The fusion method of this type has been perfected and widely adopted in the industry.

The method of transforming terrain model or ground object model has a good experience in the vision. But this method has made too many human intervention on $3 \mathrm{D}$ models, which has changed the original size of the model. At the level of meticulous mapping, the accuracy of the fusion model is slightly insufficient, which needs further research through the following research.

3.2.2 Geometric Embedded Fusion Method: The geometric embedded fusion is to make the terrain model embedded in the terrain model in the form of grid. The basic idea is to embed the contour polygon of the ground object model into the irregular triangular mesh of the terrain model, so as to obtain the influence range of the polygon. Finally, the terrain in the area of polygon is reconstructed with the polygon as a constraint to achieve the integration of the ground object model and the terrain model. Scholar Zhu Juan take the road model as an example (Zhu, 2009), regard the road boundary line as one or more arbitrary polygons, after obtaining the boundary information of the road model, the road boundary line is embedded into the terrain triangulation in turn by using the topological relationship of the terrain triangulation network, making the road boundary line a triangle in the triangulation network. Finally, the terrain model and the terrain model can be fused by deleting the terrain triangle in the road boundary polygon. Yin Xiaojun and others take the architectural model as the research object (Yin, 2011), by extracting the area of the building's terrain as the scope of the terrain model, constrained conditions to develop TIN terrain model, so as to realize the ground object model and terrain model integration.

Using this method the fusion of rivers and roads, the lake features such as models tend to just do a simple triangulations, 
bending and rolling characteristic attribute of the model will be affected.

\section{CONCLUSION}

This paper summarizes the application and development of 3D data fusion in ground object model and terrain model in recent years. According to the relationship between ground object model and terrain model, the terrain model is divided into two categories: ground object model independent terrain and ground object model dependent terrain. And this paper summarizes the 3D data of each type of terrain model of the fusion methods and research progress. According to the dependence of ground object model and terrain model, the fusion method of typical terrain model and terrain fusion is generalized. The 3D model established by aerial tilt photogrammetry has been widely recognized by the market for its low cost, high efficiency, and the characteristics of the model with its true texture mapping. However, influenced by UAV types, navigation height and its photographic platform, the 3D model based on aerial tilt photogrammetry has the characteristics of inconsistency of resolution, uneven texture of model map and uneven light. How to precisely integrate such multi resolution Irregular Triangulation Network Model and keep its original real texture and light evenly, and the transition of model boundary is natural which needs further study by related scholars. With the continuous improvement of the precision of 3D digital model, the high precision fusion of 3D ground object model and 3D terrain model is also an urgent problem to be solved. The fusion of 3D model needs to rely on the fine mapping technology, and combined with the research of geographic information systems and computer vision research, and the high precision seamless fusion of multi source 3D data requires more profound research.

\section{REFERENCES}

Agrawal A, Radha Krishna M, Joshi R, 2006. Geometry-based mapping and rendering of vector data over LOD Photo textured 3D terrain models. In Proceedings of WSCG (2006).

Bo Z.Q, Zhang Yu, 2016. Application of Pictometry Oblique Photogrammetry in Changchun City Modelling. Urban Geotechnical Investigation \& Surveying, (3), pp. 93-96.

Chen B.H., Deng Lei, Duan Y.Q. 2016, Multiple model fusion in 3-D reconstruction Illumination and scale invariance. Journal of Tsinghua University (Natural Science Edition), pp. 969-973.

Chen G.J, Chen Q.G, Cui B.L, 2009. Modelling and Rendering of Surface Targets and Terrain based on LOD. System Simulation, (S1), pp. 99-102.

Chen Q.G, 2009. Modeling and Rendering of Surface Targets and Terrain Based on LOD. China University of Petroleum (East China).

Ding Y.H, Qiu D.W, Wang F.L, 2010. Building 3D Model Reconstruction Based on Terrestrial 3D Laser Scanning Data. Bulletin of Surveying and Mapping, (3), pp. 55-57.

Gong Zhen. 2006, Research on the 3D Terrain Model for Construction and Visual Simulation. Huazhong University of Science and Technology.
Geng Z.Y, Renna, Li Y.C, 2016. Research on the fusion method of 3D models of oblique photography and large scene terrain. Science of Surveying and Mapping, 41 (11), pp. 108-113.

Hu L.J, 2013. Research on the Integration Method of 3dimentional Terrain Model and Ground Feature Model based on Level of Detail. LanZhou JiaoTong University.

Li Lin, Cheng Y.X, 2011. Research and application of fusion method between three-dimensional terrain and object. Computer Engineering and Design, 32 (12), pp. 4151-4154.

Liu X.B, 2014. Research on the fusion method of 3-dimentional feature models and multi-scale terrain. Chinese Academy of Surveying and Mapping Science.

Li Qiang, Jiang Y.M, 2008. A new mesh fusion method for terrain and terrain. Computer Engineering and Application, 44 (12), pp. 207-209.

Liu Ning, Xu Min, 2004. Research on the algorithm of terrain and terrain matching in virtual terrain modelling. Surveying and Mapping Science and technology, 21 (3), pp.215-218.

Liu Ning, 2004. Fusion algorithm and accuracy evaluation of terrain and terrain in virtual landscape simulation. The PLA Information Engineering University.

Lai L.L, Xu M.Z, Li Ming, 2007. 3d modeling of building with high precision based on photogrammetry. Journal of Geomatics, 32 (5), pp. 24-27.

Li X.W, Deng Fei, Li X.F, 2017. A Visualization Plan for Large-scale 3D Models with Oblique Photogrammetry Technology. Survey Bulletin, (4), pp. 39-43.

Polis M F, Gifford S J, Jr M K, 1995. Automating the Construction of Large-Scale Virtual Worlds. IEEE Computer Society Press.

Song H.C, Wei Y.M, Wu L.D, 2003. Matching Research of 3D Object Model with Terrain in Virtual Environment. Journal of Computer-Aided Design \& Computer Graphics, 15 (9), pp. 1167-1171.

Schilling A, Zipf A, 2003. Generation of VRML city models for focus based tour animations: integration, modeling and presentation of heterogeneous geo-data sources. Proceeding of the eighth international conference (2003), pp.39.

Sun Y.P, Fan Y.B, Hao Rui , 2015. Study of Development and Application Based Oblique Photogrammetry Technology and Constructing Real-scene 3D Productions. Geomatics \& Spatial Information Technology, 38 (11), pp. 152-154

Sun Y.Y, 2016. Application of virtual measurement in unmanned aerial vehicle surveying and mapping. Bulletin of Surveying and Mapping (7), pp. 148-149.

Wan Gang, Xia Qing, Chen Gang, 2001. The Technique of Terrain Features' Geometry Modeling Used in Virtual Terrain Environment. System Simulation, (S2), pp.73-76.

Wang G.X, Liu Ning, Wan Gang, 2005. A Fusion Algorithm and Accuracy Estimate between Road and Terrain in Virtual Terrain 
Environment, Journal of Surveying and mapping, 34 (4), pp.337-342.

Wang Hao, 2006. Research Based on terrain and terrain and Ground Feature Merging Method in virtual landform simulation. Jilin University.

Wan Gang, Chen Gang, 2002. Research on Geometry Modeling of Terrain Features in Virtual City. Journal of Surveying and mapping, 31 (1), pp.60-65.

Weber A, Benner J, 2001. Interactive Generation of Digital Terrain Models Using Multiple Data Sources. International Symposium on Digital Earth Moving. Springer-Verlag, pp.6064.

Wang A.A, Zhang Jin, 2012. Matching Method of Entity Model and Terrain Model on Buildings and Roads in Threedimensional GIS. Bulletin of Surveying and Mapping, (6), pp.84-86.

Wang S.M, 2017. Application of 3D laser scanning technology in the surveying and Mapping Engineering. Sichuan cement, (01), pp. 108.

Yin X.J, Zhao Q.Z, N.C, 2011. Research and realization of city virtual geographic environments. Computer applications and software, 28 (5), pp. 91-93.

Zhu Juan, Xu X.L, 2009. Research of the seamless fusion algorithm between road model and terrain model based on TIN. Control \& Automation, 25 (24), pp. 146-148.

Zhang J.Z, Lin Si, Li Y.F , 2016 . Comparative Analysis of AutoCAD and 3DMAX in 3D Modelling of Hydropower Station. Value Engineering, 35 (22), pp. 107-109. 